\title{
Conceptual and Instrumental Progress in Dissolution DNP
}

\author{
Fabian Jähnig ${ }^{1}$, Grzegorz Kwiatkowski ${ }^{1,2}$, and Matthias Ernst ${ }^{1 *}$ \\ ${ }^{1}$ Physical Chemistry, ETH Zürich, Vladimir-Prelog-Weg 2, 8093 Zürich, Switzerland \\ ${ }^{2}$ Institute for Biomedical Engineering, University and ETH Zürich, Gloriastrasse 35, \\ 8092 Zürich, Switzerland
}

Corresponding author e-mail: maer@ethz.ch 


\begin{abstract}
We discuss conceptual and instrumental progress in dissolution DNP since its introduction in 2003. In our view there are three critical steps in the dissolution DNP process: (i) The achievable polarization level in a sample. (ii) The time required to build up the polarization. (iii) The transfer of the sample to the measurement system with minimum loss of polarization. In this review we describe in detail these steps and the different methodological and instrumental implementations, which have been proposed to optimize them.
\end{abstract}




\section{$1 \quad$ Introduction}

The quest for generally applicable methods to enhance the equilibrium polarization beyond the Boltzmann factor has a long history in NMR research, starting with the idea of Albert Overhauser [1] to utilize electron polarization in order to increase the nuclear polarization, a concept which was later experimentally implemented by Carver and Slichter [2]. A few years later, Abragam coined the term dynamic-nuclear polarization (DNP), initially for the solid effect in diamagnetic insulators with a small concentration of paramagnetic impurities. This DNP experiment was independently proposed by Jeffries [3] and Abragam $[4,5]$. Today, the term dynamic-nuclear polarization is used in a more general way in NMR to include many methods where electron polarization is used to enhance nuclear spin polarization. For many decades, DNP was a relatively small field in NMR with limited applications due to the requirement to work at low temperatures. The early development of DNP is summarized in several books and reviews [6-8]. During the past 10-20 years, DNP became more important in mainstream NMR research mainly through two developments: (i) The steady progress in the development of cryogenic (temperatures around $100 \mathrm{~K}$ ) magicangle spinning (MAS) NMR by the group of Robert G. Griffin at MIT $[9,10]$ using a gyrotron as a high-power source for microwaves and the subsequent commercialization by BrukerBiospin. (ii) The introduction of dissolution DNP where the sample is polarized at liquidHelium temperatures using low-power microwave sources but used in the liquid state after a rapid dissolution process [11,12]. This Perspectives article will focus on the recent developments in dissolution DNP.

The principles of dissolution DNP are quite simple (see Fig. 1) [13]. The liquid sample is doped with a free radical, frozen into a glass, placed in a polarizing field (typically $3.4 \mathrm{~T}$ ) at low temperature (typically 1-2 K) and irradiated with low-power microwaves (typically 10$200 \mathrm{~mW}$ ) to build up high nuclear polarization through transfer of polarization from the almost fully-polarized electrons (Fig. 2). The sample is then rapidly dissolved using a hot liquid and transferred to a measurement system, which can either be a NMR magnet for solution-state NMR experiments or a MRI scanner for in-vivo spectroscopy or imaging. By nature of the procedure, such an approach produces a one-time polarization that has to be used efficiently. Therefore, maximizing the polarization as well as minimizing the build-up time 
and relaxation losses during the dissolution and transfer process have been important research topics during the past 10 years. The limiting step for repeated measurements is the time required to build up the polarization, which is typically in the order of $30 \mathrm{~min}$ to several hours. Therefore, methodological and hardware development for dissolution DNP has focused on implementing faster repetition rates for consecutive dissolutions, higher achievable polarizations, and on reducing magnetization loss during and after dissolution. The final step, i.e., removing the radicals from the solution, applies mostly to the in-vivo use of the hyperpolarized samples. It has, however, also some importance for spectroscopic applications, since relaxation times will be shortened by the radicals in the solution.

Typical samples for dissolution DNP make use either of trityl as a radical for direct ${ }^{13} \mathrm{C}$ polarization or TEMPO-based radicals (that are cheaper and more easily available) for generating ${ }^{1} \mathrm{H}$ polarization potentially followed by a polarization-transfer step to ${ }^{13} \mathrm{C}$ before dissolution. The driving force for the continued development of dissolution DNP is clearly the in-vivo use of highly-polarized metabolites with a strong emphasis on $1-{ }^{13} \mathrm{C}$-pyruvic acid. There are also challenging and interesting applications in high-resolution NMR spectroscopy [14] that can be addressed by dissolution DNP but they are currently a relatively minor part of the dissolution-DNP applications. Among these, application of hyperpolarized media for dynamic study of chemical reactions [15] including protein folding [16] or drug screening [17] were shown to be very promising.

In the following we will describe and discuss the different methodological and instrumental implementations, which have been introduced to optimize the critical steps in dissolution DNP. Section 2 illustrates attempts to boost the polarization levels in the sample. In Section 3 we describe approaches to reduce the time required to build up the polarization. Section 4 discusses the developments related to minimizing the loss of polarization during dissolution and transfer of the sample to the measurement system. Finally, in Section 5 we discuss techniques related to removing the radical from the solution. 


\section{Boosting Polarization Levels}

The first dissolution DNP setup [11,18] was build around a magnet charged to $3.35 \mathrm{~T}$ due to the ready availability of microwave sources at $94 \mathrm{GHz}$ (W band in EPR) with maximum powers in the $100-400 \mathrm{~mW}$ range. At the typical temperature of around $1.4 \mathrm{~K}$, the electron polarization at this field is $93 \%$ (see Fig. 2) while the ${ }^{1} \mathrm{H}$ and ${ }^{13} \mathrm{C}$ polarizations are below 1\%. A very similar hardware setup was used in the first commercial polarizer ("Hypersense") from Oxford Instruments. This principal design was copied and extended also by several home-built systems [19-21] and a prototype design for a polarizer with sterile fluid path [22]. The commercial SPINlab polarizer from GE operates at $5 \mathrm{~T}(140 \mathrm{GHz} \mathrm{EPR}$ frequency). There are also systems operating at lower static magnetic fields (2.9 T) [23] but the general trend is towards higher static magnetic fields [24-28] with the intent to increase the maximum achievable polarization. There are also designs for DNP polarizers without dissolution capability [29,30] but with more elaborate NMR and EPR measurement options for detailed investigation of mechanistic questions.

The published polarization data indicate an increase in achievable polarization as a function of the static magnetic field for direct ${ }^{13} \mathrm{C}$ polarization using trityl as a radical and pyruvate as a target molecule. Under optimum conditions polarization levels around 40-50\% are achievable in the solid state using 3.5 T polarizers [20,31]. At 4.6 T static magnetic field, pyruvate polarization levels up to $70 \%$ have been reported [24,32]. Additionally, different types of mono- [33] and bi-radicals [32] have been used to directly polarize ${ }^{13} \mathrm{C}$ in pyruvic acid but all of them exhibited inferior performance in dissolution DNP systems compared to OX063 trityl. To the best of our knowledge, there are no data available for trityl/pyruvatebased samples at higher than $5 \mathrm{~T}$ static magnetic fields with respect to dissolution DNP. A drawback of the higher fields is the significantly slower build up of the polarization at $4.6 \mathrm{~T}$ compared to $3.4 \mathrm{~T}$, a trend which seems to be even more pronounced when going to even higher $B_{0}$ fields.

The alternate route is the generation of proton polarization using TEMPO as a radical with possibly subsequent cross polarization to ${ }^{13} \mathrm{C}$ [34-40] before dissolution. In this case there is a clear dependence of the achievable polarization levels on the static magnetic field. 
At $3.4 \mathrm{~T}$ using TEMPO as a radical, proton polarization levels around $35 \%$ have been reported [35,37], while at higher magnetic fields $(6.7 \mathrm{~T})$, the achieved proton polarization levels were up to $91 \%$ [26]. In subsequent publications, typical proton polarization levels of $50-70 \%$ were reached $[40,41]$. Since the emphasis of TEMPO-based implementations of dissolution DNP is more on the faster build up and not so much on the maximum achievable ${ }^{13} \mathrm{C}$ polarization, we will discuss this in more detail in Section 3. For an overview, typical achievable polarization levels are summarized in Table 1.

Given the currently obtainable ${ }^{13} \mathrm{C}$ polarization levels of $40 \%$, and higher, and the fact that the upper limit of $100 \%$ cannot be achieved, other strategies aimed at obtaining higher signal strength have also to be considered. One approach which has been suggested recently is the use of larger-volume samples $[42,43]$. Typical dissolution DNP samples have a volume of 20-90 $\mu \mathrm{l}$ which is then diluted by the dissolution process to $4-10 \mathrm{ml}$. Sample sizes of up to $1500 \mu \mathrm{l}$ have been successfully polarized and dissolved. The applicability of this approach, however, depends strongly on the intended use after dissolution. For in-vivo imaging or localized spectroscopy in small rodents, the amount of solution that can be injected is limited and larger volume samples will give no advantage. In larger animals or human applications, however, such methods can be very important to boost the available total signal-to-noise ratio.

We note also that the large distribution in the values of the polarization level obtained under very similar conditions indicates that the accurate measurement of absolute polarization in the solid state is not trivial. At typical dissolution DNP temperatures below $1.5 \mathrm{~K}$, the ${ }^{13} \mathrm{C}$ relaxation times are estimated to be several $1000 \mathrm{~s}$. While it is easily possible to measure DNP-enhanced signals in a single shot using small-flip-angle pulses, measuring the equilibrium (Boltzmann) signals is difficult due to the long time required to reach the thermal equilibrium after cooling down the sample. Therefore, often thermal-equilibrium polarization at higher temperatures $(4.2 \mathrm{~K})$ are measured and extrapolated using the Boltzmann factors to the lower target temperature $[20,41]$. There are no systematic studies on the size of the error associated to this procedure. Another approach employs the frequency shift [24,32] of the powder line shape caused by the dipolar demagnetization field [44]. The nuclear (and electron) spins contained in the sample generate a dipolar field, which changes significantly for the polarization levels obtainable under dissolution DNP conditions. One can calculate the 
polarization-dependent shift based on equations given by Levitt for an infinitely long cylindrical sample [44] as

$$
v^{(1)}=-\frac{\mu_{0}}{4 \pi} p^{(1)} \frac{\hbar \gamma^{(1)} \gamma^{(2)} c^{(2)}}{3}
$$

where $p^{(1)}$ is the polarization and $v^{(1)}$ the frequency shift of spin $1, \gamma^{(i)}$ is the gyromagnetic ratio of spin $i$, and $c^{(2)}$ is the concentration of spin 2 in units of number of spins per $\mathrm{m}^{3}$. Assuming a ${ }^{13} \mathrm{C}$ concentration in $1-{ }^{13} \mathrm{C}$-pyruvic acid (we assume a density of $1.49 \mathrm{~g} / \mathrm{ml}$ ) of $10^{28}$ atoms $/ \mathrm{m}^{3}$ leads to a shift of the line by about $160 \mathrm{~Hz}$ for a fully polarized sample. At the same time, however, the electrons and, potentially, the protons in the sample can also lead to a shift of the line. For a radical concentration of $15 \mathrm{mmol} / 1$ a shift of about $-380 \mathrm{~Hz}$ for fully polarized electrons is calculated, while the protons in pyruvic acid give rise to a maximum shift of about $2550 \mathrm{~Hz}$. This shows that using the dipolar-demagnetization field to determine the polarization levels requires a very precise measurement of the center of gravity (first moment) of the complex powder line shape, keeping in mind that the line width of pyruvic acid is roughly $6 \mathrm{kHz}$ [32]. In our opinion, the availability of a fast and reliable method to determine the absolute polarization would be highly beneficial in order to avoid large discrepancies and errors on the quoted polarization levels in the solid state and allow fair comparison between literature data.

\section{$3 \quad$ Boosting Polarization Build Up}

Since the achievable polarization of $40 \%$ and above are already quite high and close to the upper limit of $100 \%$ as discussed above, a faster build up of the polarization or the generation of polarization in several samples in parallel has become an important option to shorten the repetition time between experiments. Table 1 summarizes some typical build-up times reported in the literature.

The problem of slow polarization build up is especially pronounced for low- $\gamma$ nuclei, e.g. ${ }^{13} \mathrm{C}$ or ${ }^{15} \mathrm{~N}$ and at higher magnetic fields. Several researches reported that adding a small quantity (on the order of 1-4 mM) of a $\mathrm{Gd}^{3+}$ chelate can significantly speed-up the build-up 
time by increasing the electronic relaxation of the spin system. The achievable polarization level is also usually enhanced. Moreover, if the commercial, Gd-based MRI relaxation agents are used, there is no need for an additional filtration step [45,46] in case the solution is intended for an in-vivo application.

For faster polarization build up, two approaches have been proposed and experimentally implemented. One approach to faster ${ }^{13} \mathrm{C}$ polarization build up uses an indirect way to generate ${ }^{13} \mathrm{C}$ polarization by using DNP to enhance the ${ }^{1} \mathrm{H}$ polarization and, in a second step, to transfer this polarization to the ${ }^{13} \mathrm{C}$ (or other) nuclei using cross polarization (CP) [34-40]. In MAS DNP [9,10] CP is routinely used to transfer polarization from ${ }^{1} \mathrm{H}$ to lower-frequency nuclei like ${ }^{13} \mathrm{C} /{ }^{15} \mathrm{~N}$. The implementation of $\mathrm{CP}$ requires a double-tuned NMR circuit in the DNP probe that allows reasonably high radio-frequency (rf) fields (at least 40$50 \mathrm{kHz}$ ) and is compatible with dissolution. Adiabatic half-passage pulses can be used instead of hard pulses to avoid projection losses due to broad lines and limited rf-field amplitudes at the start and the end of the cross-polarization period [37,39]. Multiple contact time CP [38] allows a further boost of the obtainable ${ }^{13} \mathrm{C}$ polarization. Using $\mathrm{CP}$ allows a build up of the ${ }^{13} \mathrm{C}$ polarization with the time constant of the ${ }^{1} \mathrm{H}$ polarization build up and allows the use of cheaper and more readily available radicals like TEMPO. The speed up of the polarization build-up time depends on the details of the sample composition but increases of up to a factor of five (Fig. 3) have been observed. The main obstacle for a more widespread use of TEMPObased DNP in combination with cross polarization for in-vivo imaging applications is the problem of combining pyruvic acid with TEMPO radicals. For spectroscopic in-vivo application TEMPO based samples have been used, although without employing the CP techniques [47]. The use of cross polarization opens up the possibility to polarize other nuclei than ${ }^{13} \mathrm{C}$ using the same principal setup and sample composition. This has recently been demonstrated for quadrupolar nuclei like ${ }^{6} \mathrm{Li}[40]$.

By frequency modulation of the microwaves irradiation $[41,48]$ a better saturation of broad radical lines in TEMPO-based samples can be achieved. Especially at lower radical concentration, a significant reduction of the build-up time constant could be observed while at the same time higher polarization levels were achieved. The acceleration of the ${ }^{1} \mathrm{H}$ polarization build up depends very strongly on the sample composition (radical 
concentration), on the exact irradiation frequency, and on the temperature of the sample. At $1.2 \mathrm{~K}$ [41] and $50 \mathrm{mM}$ TEMPO concentrations, a factor of 2-3 faster build up was observed with about 50\% higher polarization levels (Fig. 4). To the best of our knowledge no results for frequency modulation of microwave irradiation has been published for trityl-based samples and it is not clear whether any enhancements can be expected given the relatively narrow trityl EPR lines.

Even using all the methods discussed so far, polarization build-up times are still significantly longer than the desired repetition rates for in-vivo MRI experiments. This gap can be closed by using multi-sample systems [20-22,49] where multiple samples can be loaded into the cryostat and polarized either sequentially or in parallel. There have been two designs proposed, one that uses a sample changer (revolver design) in the liquid Helium bath [20] (Fig. 5a) and another one, developed and implemented independently by several groups, that allows the use of several (3-4) independent sample holders and dissolution systems $[21,22,49]$ in a single cryostat (Fig. 5b). Figure 5 shows schematic drawings of the cryogenic inserts of two of the designs [20,49]. The revolver design allows the loading of a maximum of six samples, which can be polarized and dissolved sequentially. This approach solves mainly the problem of loading and unloading of samples during experimental runs. In principle, the samples can also be polarized simultaneously, but in the published design dissolution requires pressurizing the cryostat, thus leading to an increase in temperature of the liquid Helium with a concomitant loss of polarization of the other samples. The second design relies on separate sample holders, which allow the simultaneous polarization and very fast sequential dissolution of all samples due to the sealing of the dissolution space from the Helium atmosphere. This permits dissolution of a sample without pressure/temperature changes in the main Helium dewar. Successive dissolutions can be done within a few minutes [49]. The polarization levels achievable in such multi-sample systems seem to be slightly lower than the ones achieved in dedicated single-sample polarizers, mainly due to difficulties in the delivery of microwave power to multiple samples. 


\section{$4 \quad$ Low-Loss Transfer of Polarized Samples}

The last important step in dissolution DNP is the fast and "lossless" transfer of polarization to a NMR spectrometer or MR scanner. Usually this transfer is achieved as an integral part of the dissolution procedure where hot solvent is used to dissolve the frozen and polarized sample [11]. High-pressure Helium gas is used to flush the solution out of the magnet and transfer it through tubing to the measurement system. At the end it can either be collected in a NMR sample tube for solution-state NMR measurements or in a syringe for injection into an animal. There are three critical phases in dissolution and transfer of the polarized solution: (i) Lifting of the solid sample out of the liquid-Helium bath and preparation for dissolution. (ii) Dissolution and transfer of the liquid out of the magnet and into the sample collector. (iii) Settling of the sample in the NMR spectrometer to reduce susceptibility broadening or quality control and injection into animal without air bubbles.

The transfer of the sample out of the liquid Helium bath is usually achieved by lifting the sample cup up with a grabber. If the sealing of the dissolution system to the sample container is fully gastight, dissolution can be achieved without pressurizing the cryostat. Such a design speeds up the dissolution procedure and prevents the temperature of the liquid Helium bath in the cryostat to increase significantly, which is mostly important for multisample polarizers. It is critical to prevent heating up of the solid sample after lifting it out of the liquid Helium bath since ${ }^{13} \mathrm{C}$ relaxation in the solid can increase significantly at higher temperatures.

After dissolution, the sample is liquid and solution-state NMR relaxation times determine the loss of polarization. It is well known that at low fields, relaxation times increase and the loss of polarization is accelerated [50]. The transfer from the polarizer magnet to the destination system will have a certain magnetic-field profile, which depends on the relative field direction and the distance of the two magnets (see Fig. 6). Polarization losses due to enhanced relaxation at low magnetic fields can be reduced by using a magnetic tunnel [51] (Fig. 6) built from small permanent magnets. In such a tunnel, the field is always above a certain value reducing the relaxation losses during the shuttle time. Another contribution to the solution-state NMR relaxation properties comes from the presence of the free radicals in 
the solution. It is, therefore, advisable to remove the radical from the solution rapidly after dissolution. This is discussed in more detail in the next section.

The use of singlet states [52] instead of Zeeman polarization for the transfer process can lead to significantly increased relaxation times. This approach has also been used in combination with dissolution DNP to extend the time scale during which the polarization can be used $[17,53]$. We will not discuss this approach in more detail.

After the transfer of the dissolved sample into a NMR sample tube, small gas bubbles and turbulences of the liquid sample are formed, causing line broadening in NMR experiments [54]. Sample motion effects can be quantified using diffusion measurements which in turn can be used to optimize the parameters for the dissolution such that gas bubbles and sample motion are minimized. Automatic sample injection systems [55,56] have been designed that prevent the formation of gas bubbles and allow kinetic studies by dividing the hyperpolarized sample into multiple batches. A flow injection system [57] can be used to minimize the turbulences in the sample tube using a high-pressure liquid instead of gases to inject the sample into the NMR sample tube. For in-vivo experiments, the sample is usually transferred to a syringe which is then used to inject the hyperpolarized substrate into the animal. There are also automatic injection systems for in-vivo applications [58].

Alternate setups for dissolution and sample transfer have been also proposed. The probably most elegant setup for NMR spectroscopic investigations is the two-center magnet [59] where polarization (3.4 T) and NMR measurements (9.4 T) happen in the same magnet in two different areas with different static magnetic fields (Figure 7). This setup offers the advantage that the transfer ways are very short with concomitant low relaxation loss. The sample is transferred by an external actuator from the polarizing field to the dissolution system near the measurement field in the solid state and can be dissolved by a heated liquid and flow into the NMR sample tube. For microfluidics applications, a transfer system using a linear actuator to transfer the sample between a freezing zone where polarization is build up to a rapid melting zone and then on to a NMR detection circuit [60] has been proposed and implemented. For very slowly relaxing samples like some silicon nano- and microparticles, a manual transfer of the cold solid sample out of the magnet to the measurement magnet is also 
feasible [61]. The same considerations as discussed above for the dissolution setup using a hot liquid are also valid for all these alternate methods.

\section{Removing the Radicals}

There are two reasons that suggest removing the radical from the solution during or after dissolution: (i) Especially at higher radical concentrations as used in TEMPO-based dissolution DNP samples, the radical can increase the relaxation times of the hyperpolarized sample in the solution [50]. (ii) For in-vivo use, the radical should be removed from the sample because of its potential toxicity. This is especially relevant for clinical applications of hyperpolarized MRI. For trityl-based pyruvate samples, changing the $\mathrm{pH}$ to a low value leads to an aggregation of the radical which can then be removed using a simple cotton filter [22]. This method is implemented in the commercial SPINlab polarizer. Another solution proposed to this problem in TEMPO-based samples is the use of sodium ascorbate (vitamin C) as a scavenger in the dissolution process $[62,63]$. The radical will be reduced and the ascorbyl radicals rapidly disproportionate leaving no free radicals in the solution.

A very elegant way of preventing radicals from being present in the solution is the use of hybrid organosilica materials which are prepared by a Sol-Gel process using a template [64] that contain chemically bound radicals. The target substance is put into the cavities of the organosilica material and polarized in the usual way. After dissolution, the solid organosilica material can be filtered out in the polarizer magnet using a cotton filter, thus leading to a radical-free solution. Using a very different approach, Eichhorn et al. [65] showed that enough radicals are formed in a neat pyruvate acid solution after irradiating it with UV light at low temperatures. Dynamic polarization of such a sample leads to steady-state polarization levels of around $10 \%$. This optical doping of a sample has subsequently been applied to invivo spectroscopic studies. Nonetheless, at the current stage, the intrinsically low achievable polarization prevents this method to be widely applicable.

Recently, the synthesis of a modified form of the BDPA radical was reported [66]. By attaching a benzyl alcohol group, its solubility in neat pyruvic acid can be significantly 
increased allowing for efficient polarization of ${ }^{13} \mathrm{C}$ nuclei, while still keeping it insoluble in the aqueous solution. After dissolution, the radical sediments out of the solution and can be easily removed with a cotton filter during the shuttling to the detection system. Finally, an alternative approach was presented [67] where the dissolution solvent was composed of a immiscible mixture of aqueous and organic solvent vapors. By assuring that one of the solvent is preferential in dissolving the polarized material and the other one in dissolving the exogenous radical, the paramagnetic adduct can be conveniently separated from the polarization target and consequently removed. In addition, due to phase separation between the solvents, the effective dilution volume of the polarization product is significantly reduced. 


\section{Outlook and Conclusions}

The progress in hardware and methodology for dissolution DNP resulted in a very high level of achievable nuclear spin polarization. This is especially true for ${ }^{1} \mathrm{H}$ and ${ }^{13} \mathrm{C}$ nuclei in selected compounds with long relaxation times. As a result, the focus point of research has shifted towards decreasing the polarization build-up time and preserving the high polarization level in the liquid state. Nevertheless, more effort is necessary to improve the polarization

efficiency for other low- $\gamma$ nuclei, e.g., ${ }^{15} \mathrm{~N},{ }^{6} \mathrm{Li}$, and others that might constitute interesting biological probes. Accelerating the polarization build up is clearly a research field where improvements and novel implementations are required. This might require synthesis of new radicals with enhanced physical-chemical properties (e.g. relaxation times) which are tuned to external conditions (e.g., magnetic field or temperature) or new approaches regarding the transfer of polarization. Another possible way to improve DNP further is to generate higher polarization levels at higher temperatures. If the same levels of polarization that are currently available at $1.5 \mathrm{~K}$ could be achieved at $4.2 \mathrm{~K}$ this would simplify the design of polarizers significantly. Most likely such a shift to higher temperatures would require higher static fields to achieve the same electron polarizations and the use of different radicals.

\section{Acknowledgements}

We would like to thank Sebastian Kozerke and his group from the Institute of Biomedical Engineering at ETH Zurich for the good and fruitful collaboration in the field of dissolution DNP and Beat H. Meier, Kazuyuki Takeda, Michael Batel, Marcin Krajewski, and Gunnar Jeschke for continued discussion and support. Andreas Hunkeler and Alexander Däpp provided excellent support for the design and construction of the mechanical and electrical parts of the DNP systems built in our laboratory. This work was supported by the ETH Zurich (Grant ETH-14 06-1) and the Swiss National Science Foundation (Grant 200021_149707). 


\section{References}

[1] A. Overhauser, Polarization of nuclei in metals, Phys. Rev. 92 (1953) 411-415.

[2] T.R. Carver, C.P. Slichter, Experimental verification of the Overhauser nuclear polarization effect, Phys. Rev. 102 (1956) 975-980.

[3] C.D. Jeffries, Polarization of nuclei by resonance saturation in paramagnetic crystals, Phys. Rev. 106 (1957) 164-165.

[4] A. Abragam, W.G. Proctor, A new method of dynamic polarization of atomic nuclei in solids, Compt. Rend. 246 (1958) 2253-2256.

[5] A. Abragam, W.G. Proctor, Spin temperature, Phys. Rev. 109 (1958) 1441-1458.

[6] M. Goldman, Spin temperature and nuclear magnetic resonance in solids, 1970.

[7] A. Abragam, M. Goldman, Principles of Dynamic Nuclear-Polarization, Rep. Prog. Phys. 41 (1978) 395-467.

[8] R.A. Wind, M. Duijvestijn, C. van der Lugt, A. Manenschijn, J. Vriend, Applications of Dynamic Nuclear-Polarization in C-13 Nmr in Solids, Prog. NMR Spectr. 17 (1985) 33-67.

[9] M.L. Mak-Jurkauskas, R.G. Griffin, High- Frequency Dynamic Nuclear Polarization, John Wiley \& Sons, Ltd, 2010. doi:10.1002/9780470034590.emrstm1183.

[10] V.K. Michaelis, T.-C. Ong, M.K. Kiesewetter, D.K. Frantz, J.J. Walish, E. Ravera, et al., Topical Developments in High-Field Dynamic Nuclear Polarization, Israel J. Chem. 54 (2014) 207-221. doi:10.1002/ijch.201300126.

[11] J.-H. Ardenkjær-Larsen, B. Fridlund, A. Gram, G. Hansson, L. Hansson, M.H. Lerche, et al., Increase in signal-to-noise ratio of $>10,000$ times in liquid-state NMR, Proc. Natl. Acad. Sci. USA. 100 (2003) 10158-10163. doi:10.1073/pnas.1733835100.

[12] K. Golman, J.-H. Ardenkjær-Larsen, J.S. Petersson, S. Mansson, I. Leunbach, Molecular imaging with endogenous substances, Proc. Natl. Acad. Sci. USA. 100 (2003) 10435-10439. doi:10.1073/pnas.1733836100.

[13] W. Köckenberger, Dissolution Dynamic Nuclear Polarization, eMagRes. (n.d.). doi:10.1002/9780470034590.emrstm1311.

[14] J.-H. Ardenkjær-Larsen, G.S. Boebinger, A. Comment, S. Duckett, A.S. Edison, F. Engelke, et al., Facing and Overcoming Sensitivity Challenges in Biomolecular NMR Spectroscopy, Angew. Chem. Int. Ed. Engl. 54 (2015) 9162-9185. doi:10.1002/anie.201410653.

[15] E. Miclet, D. Abergel, A. Bornet, J. Milani, S. Jannin, G. Bodenhausen, Toward quantitative measurements of enzyme kinetics by dissolution dynamic nuclear polarization, J. Phys. Chem. Lett. 5 (2014) 3290-3295. doi:10.1021/jz501411d.

[16] H.-Y. Chen, M. Ragavan, C. Hilty, Protein Folding Studied by Dissolution Dynamic Nuclear Polarization, Angew. Chem. Int. Ed. 52 (2013) 9192-9195. doi:10.1002/anie.201301851.

[17] R. Buratto, A. Bornet, J. Milani, D. Mammoli, B. Vuichoud, N. Salvi, et al., Drug Screening Boosted by Hyperpolarized Long-Lived States in NMR, ChemMedChem. 9 (2014) 2509-2515. doi:10.1002/cmdc.201402214.

[18] J. Wolber, F. Ellner, B. Fridlund, A. Gram, H. Johannesson, G. Hansson, et al., Generating highly polarized nuclear spins in solution using dynamic nuclear polarization, Nucl. Instr. and Meth. A. 526 (2004) 173-181.

[19] A. Comment, B. Van Den Brandt, K. Uffmann, F. Kurdzesau, S. Jannin, J.A. Konter, et al., Design and performance of a DNP prepolarizer coupled to a rodent MRI scan- 
[20] M. Batel, M. Krajewski, K. Weiss, O. With, A. Däpp, A. Hunkeler, et al., A multisample $94 \mathrm{GHz}$ dissolution dynamic-nuclear-polarization system, J. Magn. Reson. 214 (2012) 166-174. doi:10.1016/j.jmr.2011.11.002.

[21] Y. Crémillieux, F. Goutailler, B. Montcel, D. Grand, G. Vermeulen, P.-E. Wolf, A Super-Wide Bore DNP System for Multiple Sample Polarization: Cryogenic Performance and Polarization at Low Temperature, Appl. Magn. Reson. 43 (2012) 167180. doi:10.1007/s00723-012-0354-5.

[22] J.-H. Ardenkjær-Larsen, A.M. Leach, N. Clarke, J. Urbahn, D. Anderson, T.W. Skloss, Dynamic nuclear polarization polarizer for sterile use intent, NMR Biomed. 24 (2011) 927-932. doi:10.1002/nbm.1682.

[23] M.C. Cassidy, C. Ramanathan, D.G. Cory, J.W. Ager, C.M. Marcus, Radical-free dynamic nuclear polarization using electronic defects in silicon, Phys. Rev. B. 87 (2013). doi:10.1103/PhysRevB.87.161306.

[24] H. Johannesson, S. Macholl, J.-H. Ardenkjær-Larsen, Dynamic Nuclear Polarization of [1-13C]pyruvic acid at 4.6 tesla, J. Magn. Reson. 197 (2009) 167-175.

[25] S. Jannin, A. Comment, F. Kurdzesau, J.A. Konter, P. Hautle, B. Van Den Brandt, et al., A $140 \mathrm{GHz}$ prepolarizer for dissolution dynamic nuclear polarization, J. Chem. Phys. 128 (2008). doi:10.1063/1.2951994.

[26] S. Jannin, A. Bornet, R. Melki, G. Bodenhausen, High field dynamic nuclear polarization at 6.7 T: Carbon-13 polarization above $70 \%$ within 20 min, Chem. Phys. Lett. 549 (2012) 99-102.

[27] T. Cheng, A. Capozzi, Y. Takado, R. Balzan, A. Comment, Over 35\% liquid-state 13C polarization obtained via dissolution dynamic nuclear polarization at $7 \mathrm{~T}$ and $1 \mathrm{~K}$ using ubiquitous nitroxyl radicals, Phys. Chem. Chem. Phys. 15 (2013) 20819-4. doi:10.1039/c3ep53022a.

[28] L.L. Lumata, R.W. Martin, A.K. Jindal, Z. Kovacs, M.S. Conradi, M.E. Merritt, Development and performance of a 129-GHz dynamic nuclear polarizer in an ultra-wide bore superconducting magnet, Magnetic Resonance Materials in Physics, Biology and Medicine. 28 (2015) 195-205.

[29] A. Feintuch, D. Shimon, Y. Hovav, D. Banerjee, I. Kaminker, Y. Lipkin, et al., A Dynamic Nuclear Polarization spectrometer at $95 \mathrm{GHz} / 144 \mathrm{MHz}$ with EPR and NMR excitation and detection capabilities, J. Magn. Reson. 209 (2011) 136-141. doi:10.1016/j.jmr.2010.12.010.

[30] B.D. Armstrong, D.T. Edwards, R.J. Wylde, S.A. Walker, S. Han, A $200 \mathrm{GHz}$ dynamic nuclear polarization spectrometer, Phys. Chem. Chem. Phys. 12 (2010) 5920. doi:10.1039/c002290j.

[31] J.-H. Ardenkjær-Larsen, S. Macholl, H. Johannesson, Dynamic Nuclear Polarization with Trityls at 1.2 K, Appl. Magn. Reson. 34 (2008) 509-522. doi:10.1007/s00723008-0134-4.

[32] S. Macholl, H. Johannesson, J.-H. Ardenkjær-Larsen, Trityl biradicals and 13C dynamic nuclear polarization, Phys. Chem. Chem. Phys. 12 (2010) 5804-5817.

[33] L.L. Lumata, S.J. Ratnakar, A. Jindal, M.E. Merritt, A. Comment, C. Malloy, et al., BDPA: An efficient polarizing agent for fast dissolution dynamic nuclear polarization NMR spectroscopy, Chem. Eur. J. 17 (2011) 10825-10827.

[34] A.J. Perez Linde, Application of cross polarisation techniques to dynamic nuclear polarisation dissolution experiments, 2010.

[35] S. Jannin, A. Bornet, S. Colombo, G. Bodenhausen, Low-temperature cross polariza- 
tion in view of enhancing dissolution Dynamic Nuclear Polarization in NMR, Chem. Phys. Lett. 517 (2011) 234-236.

[36] A. Bornet, R. Melki, S. Jannin, G. Bodenhausen, Cross Polarization for Dissolution Dynamic Nuclear Polarization Experiments at Readily Accessible Temperatures 1.2, Appl. Magn. Reson. 43 (2012) 107-117.

[37] M. Batel, M. Krajewski, A. Däpp, A. Hunkeler, B.H. Meier, S. Kozerke, et al., Dissolution dynamic nuclear polarization efficiency enhanced by Hartmann-Hahn cross polarization, Chem. Phys. Lett. 554 (2012) 72-76. doi:10.1016/j.cplett.2012.10.018.

[38] A. Bornet, R. Melzi, A.J. Perez Linde, P. Hautle, B. van den Brandt, S. Jannin, et al., Boosting Dissolution Dynamic Nuclear Polarization by Cross Polarization, J. Phys. Chem. Lett. 4 (2013) 111-114. doi:10.1021/jz301781t.

[39] M. Batel, A. Däpp, A. Hunkeler, B.H. Meier, S. Kozerke, M. Ernst, Crosspolarization for dissolution dynamic nuclear polarization, Phys. Chem. Chem. Phys. 16 (2014) 21407-21416. doi:10.1039/c4cp02696a.

[40] A.J. Perez Linde, A. Bornet, J. Milani, B. Vuichoud, R. Melzi, S. Jannin, et al., Cross polarization from $1 \mathrm{H}$ to quadrupolar $6 \mathrm{Li}$ nuclei for dissolution DNP, Phys. Chem. Chem. Phys. 16 (2014) 24813-24817. doi:10.1039/C4CP03592E.

[41] A. Bornet, J. Milani, B. Vuichoud, A.J. Perez Linde, G. Bodenhausen, S. Jannin, Microwave frequency modulation to enhance Dissolution Dynamic Nuclear Polarization Dedicated to To Martial Rey, as a token of appreciation, Chem. Phys. Lett. 602 (2014) 63-67. doi:10.1016/j.cplett.2014.04.013.

[42] A. Comment, J. Rentsch, F. Kurdzesau, S. Jannin, K. Uffmann, R.B. van Heeswijk, et al., Producing over $100 \mathrm{ml}$ of highly concentrated hyperpolarized solution by means of dissolution DNP, J. Magn. Reson. 194 (2008) 152-155.

doi:10.1016/j.jmr.2008.06.003.

[43] S. Bowen, J.-H. Ardenkjær-Larsen, Enhanced performance large volume dissolutionDNP, J. Magn. Reson. 240 (2014) 90-94. doi:10.1016/j.jmr.2014.01.009.

[44] M.H. Levitt, Demagnetization field effects in two-dimensional solution NMR, Concepts Magn. Reson. 8 (1996) 77-103. doi:10.1002/(SICI)10990534(1996)8:2<77::AID-CMR1>3.0.CO;2-L.

[45] L.L. Lumata, M.E. Merritt, C.R. Malloy, A.D. Sherry, Z. Kovacs, Impact of Gd 3+on DNP of [1- 13C]Pyruvate Doped with Trityl OX063, BDPA, or 4-Oxo-TEMPO, J. Phys. Chem. A. 116 (2012) 5129-5138. doi:10.1021/jp302399f.

[46] E. Ravera, D. Shimon, A. Feintuch, D. Goldfarb, S. Vega, A. Flori, et al., The effect of Gd on trityl-based dynamic nuclear polarisation in solids, Phys. Chem. Chem. Phys. (2015). doi:10.1039/c5cp04138d.

[47] J.A.M. Bastiaansen, T. Cheng, H. Lei, R. Gruetter, A. Comment, Direct noninvasive estimation of myocardial tricarboxylic acid cycle flux in vivo using hyperpolarized 13C magnetic resonance, J. Mol. Cell. Cardiol. 87 (2015) 129-137. doi:10.1016/j.yjmcc.2015.08.012.

[48] Y. Hovav, A. Feintuch, S. Vega, D. Goldfarb, Dynamic nuclear polarization using frequency modulation at 3.34 T, J. Magn. Reson. 238 (2014) 94-105. doi:10.1016/j.jmr.2013.10.025.

[49] M. Krajewski, M. Batel, K. Weiss, A. Sigfridsson, G. Batsios, M. Ernst, et al., A flexible multi-sample DNP system for rapid sequential dissolutions, Proc. Intl. Soc. Mag. Reson. Med. 21 (2013) 3941.

[50] P. Miéville, S. Jannin, G. Bodenhausen, Relaxometry of insensitive nuclei: Optimizing dissolution dynamic nuclear polarization, J. Magn. Reson. 210 (2011) 137-140. 
[51] J. Milani, B. Vuichoud, A. Bornet, P. Miéville, R. Mottier, S. Jannin, et al., A magnetic tunnel to shelter hyperpolarized fluids, Rev. Sci. Instrum. 86 (2015) 024101. doi:10.1063/1.4908196.

[52] M.H. Levitt, Singlet Nuclear Magnetic Resonance, Annu. Rev. Phys. Chem. (2011). doi:10.1146/annurev-physchem-032511-143724.

[53] R. Buratto, D. Mammoli, E. Chiarparin, G. Williams, G. Bodenhausen, Exploring Weak Ligand-Protein Interactions by Long-Lived NMR States: Improved Contrast in Fragment-Based Drug Screening, Angew. Chem. Int. Ed. 53 (2014) 11376-11380. doi:10.1002/anie.201404921.

[54] J. Granwehr, R. Panek, J. Leggett, W. Köckenberger, Quantifying the transfer and settling in NMR experiments with sample shuttling, J. Chem. Phys. 132 (2010) 244507. doi:10.1063/1.3446804.

[55] S. Bowen, C. Hilty, Time- Resolved Dynamic Nuclear Polarization Enhanced NMR Spectroscopy, Angew. Chem. Int. Ed. 47 (2008) 5235-5237. doi:10.1002/anie.200801492.

[56] S. Bowen, C. Hilty, Rapid sample injection for hyperpolarized NMR spectroscopy, Phys. Chem. Chem. Phys. 12 (2010) 5766-5770.

[57] H.-Y. Chen, C. Hilty, Implementation and Characterization of Flow Injection in Dissolution Dynamic Nuclear Polarization NMR Spectroscopy, ChemPhysChem. 16 (2015) 2646-2652. doi:10.1002/cphc.201500292.

[58] T. Cheng, M. Mishkovsky, J.A.M. Bastiaansen, O. Ouari, P. Hautle, P. Tordo, et al., Automated transfer and injection of hyperpolarized molecules with polarization measurement prior to in vivo NMR, NMR Biomed. 26 (2013) 1582-1588. doi:10.1002/nbm.2993.

[59] J. Leggett, R. Hunter, J. Granwehr, R. Panek, A.J. Perez Linde, A.J. Horsewill, et al., A dedicated spectrometer for dissolution DNP NMR spectroscopy, Phys. Chem. Chem. Phys. 12 (2010) 5883-5892. doi:10.1039/c002566f.

[60] M. Sharma, G. Janssen, J. Leggett, A.P.M. Kentgens, P.J.M. van Bentum, Rapid-melt Dynamic Nuclear Polarization, J. Magn. Reson. 258 (2015) 40-48. doi:10.1016/j.jmr.2015.06.007.

[61] M.C. Cassidy, H.R. Chan, B.D. Ross, P.K. Bhattacharya, C.M. Marcus, In vivo magnetic resonance imaging of hyperpolarized silicon particles, Nature Nanotech. 8 (2013) 363-368. doi:10.1038/nnano.2013.65.

[62] P. Miéville, P. Ahuja, R. Sarkar, S. Jannin, P.R. Vasos, S. Gerber-Lemaire, et al., Scavenging free radicals to preserve enhancement and extend relaxation times in NMR using dynamic nuclear polarization, Angew. Chem. Int. Ed. Engl. 49 (2010) 6182-6185.

[63] P. Miéville, P. Ahuja, R. Sarkar, S. Jannin, P.R. Vasos, S. Gerber-Lemaire, et al., Erratum: Scavenging free radicals to preserve enhancement and extend relaxation times in NMR using dynamic nuclear polarization (Angewandte Chemie - International Edition (2010) (49), Angew. Chem. Int. Ed. Engl. 49 (2010) 7834.

[64] D. Gajan, A. Bornet, B. Vuichoud, J. Milani, R. Melzi, H.A. van Kalkeren, et al., Hybrid polarizing solids for pure hyperpolarized liquids through dissolution dynamic nuclear polarization, Proc. Natl. Acad. Sci. USA. 111 (2014) 14693-14697. doi:10.1073/pnas.1407730111.

[65] T.R. Eichhorn, Y. Takado, N. Salameh, A. Capozzi, T. Cheng, J.-N. Hyacinthe, et al., Hyperpolarization without persistent radicals for in vivo real-time metabolic imaging, 
Proc. Natl. Acad. Sci. USA. 110 (2013) 18064-18069.

doi:10.1073/pnas.1314928110.

[66] J.L. Muñoz-Gómez, E. Monteagudo, V. Lloveras, T. Parella, J. Veciana, J. VidalGancedo, A benzyl alcohol derivative of the BDPA radical for fast dissolution dynamic nuclear polarization NMR spectroscopy, Org. Biomol. Chem. 13 (2015) 26892693. doi:10.1039/c4ob02356k.

[67] T. Harris, C.O. Bretschneider, L. Frydman, Dissolution DNP NMR with solvent mixtures: Substrate concentration and radical extraction, J. Magn. Reson. 211 (2011) 96100. doi:10.1016/j.jmr.2011.04.001.

[68] T.A. Siaw, M. Fehr, A. Lund, A. Latimer, S.A. Walker, D.T. Edwards, et al., Effect of electron spin dynamics on solid-state dynamic nuclear polarization performance, Phys. Chem. Chem. Phys. 16 (2014) 18694-18706. doi:10.1039/c4cp02013h. 


\section{Figure Captions}

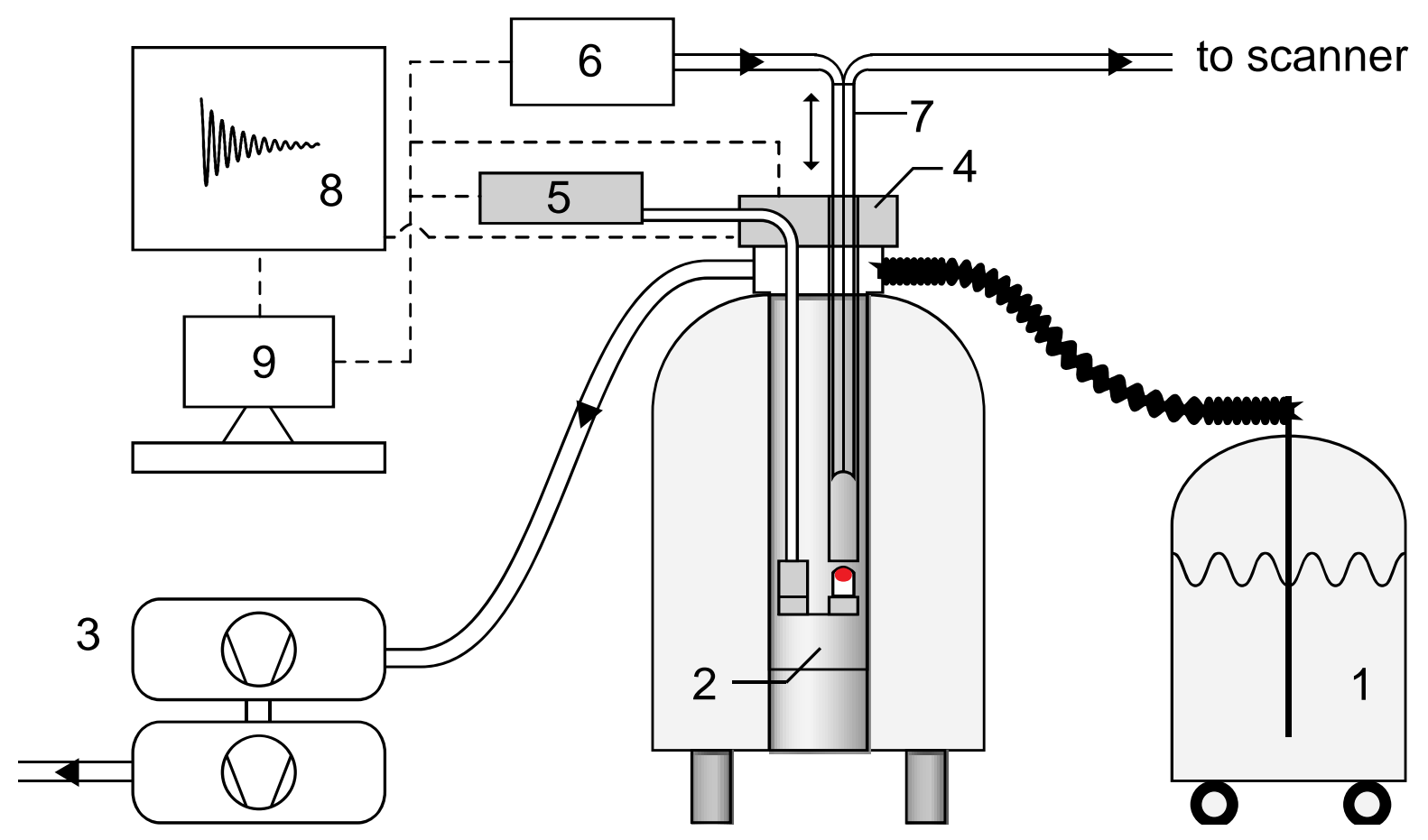

Figure 1: Schematic drawing of the complete dissolution DNP system. A flow-type cryo system consists of an external liquid-Helium supply dewar (1) connected to the variabletemperature cryostat (2) via a vacuum shielded transfer line. Helium is dragged into the sample space by evacuating the cryostat with two vacuum pumps (3) connected in series. The DNP probe (4) is mounted and sealed to the cryostat within the bore of the magnet. A microwave source (5) is connected to the waveguide leading to the sample space. The dissolution system (6) is attached to the tubing of the dissolution stick (7) for dissolution and shuttling to the nearby NMR/MRI spectrometer. An NMR console (8) is used to monitor the nuclear polarization while the entire system is controlled by computer software (9). Dotted lines indicate data communication pathways. Copied with permission from [20]. 


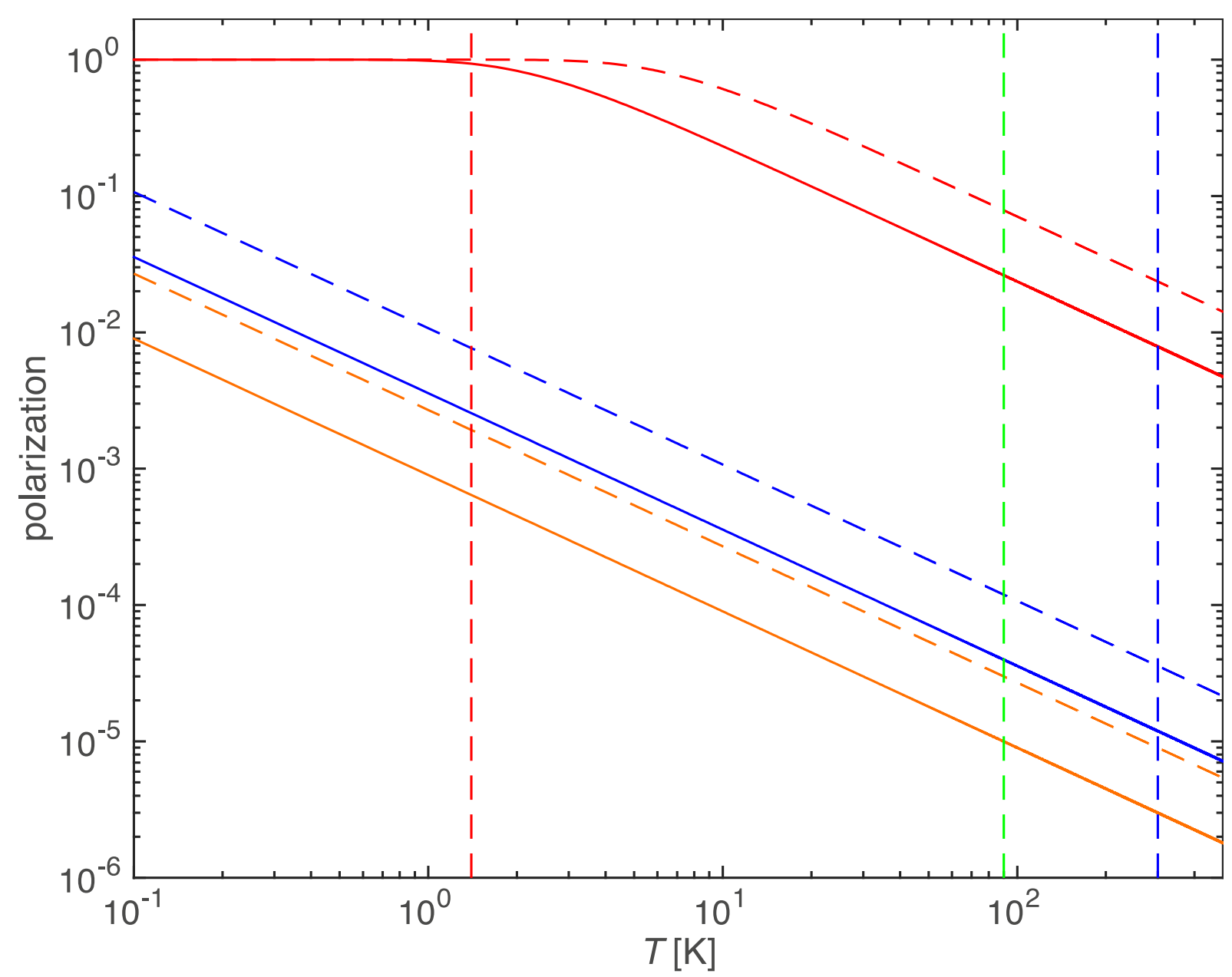

Figure 2: Plot of electron (red), ${ }^{1} \mathrm{H}$ (blue), and ${ }^{13} \mathrm{C}$ (orange) polarization as a function of temperature for static magnetic fields of $3.5 \mathrm{~T}$ (solid lines) and 10.5 $14 \mathrm{~T}$ (dashed lines). The vertical dashed lines correspond to temperatures of $1.5 \mathrm{~K}$ (red), $90 \mathrm{~K}$ (green) and $300 \mathrm{~K}$ (blue). 


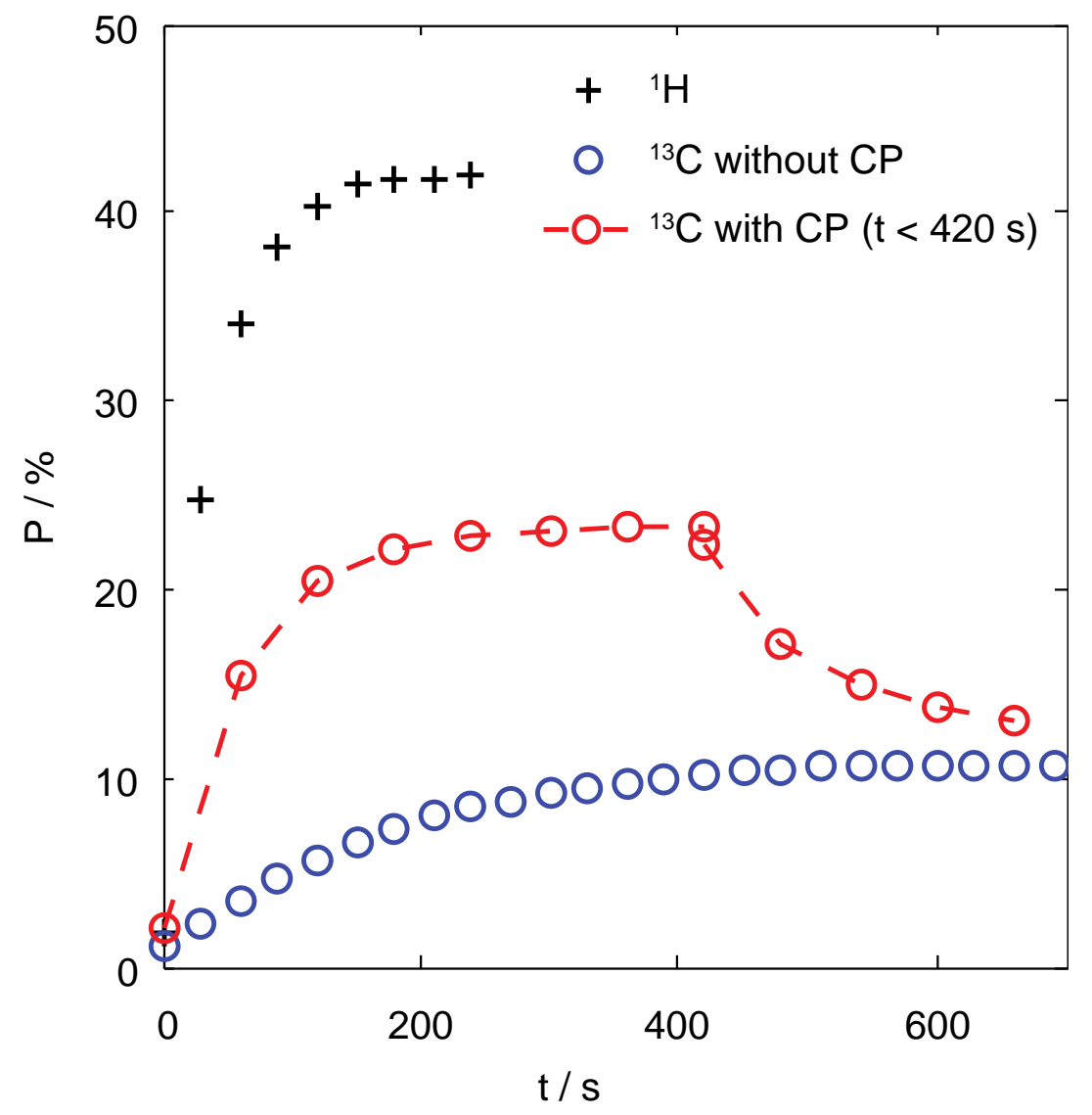

Figure 3: Solid-state DNP and DNP-CP polarization levels of $3.5 \mathrm{M}\left[1,4-{ }^{13} \mathrm{C}\right]$ fumaric acid in DMSO-d6 doped with $40 \mathrm{mM}$ TEMPO and $16 \mathrm{mM}$ trityl. The DNP polarization build-up times are $\tau_{1 \mathrm{H}}=36.3 \mathrm{~s}$ and $\tau_{13 \mathrm{C}}=181.3 \mathrm{~s}$, the DNP-CP build-up time using multiple CP pulses during polarization is $\tau_{1 \mathrm{H}}=60.0 \mathrm{~s}$ and the thermal-mixing time $T_{\mathrm{TM}}=82.0 \mathrm{~s}$. The thermalmixing time $T_{\mathrm{TM}}$ describes the decay towards the DNP equilibrium after the last CP pulse at $t$ $=420 \mathrm{~s}$. Copied with permission from [37]. 
(a)

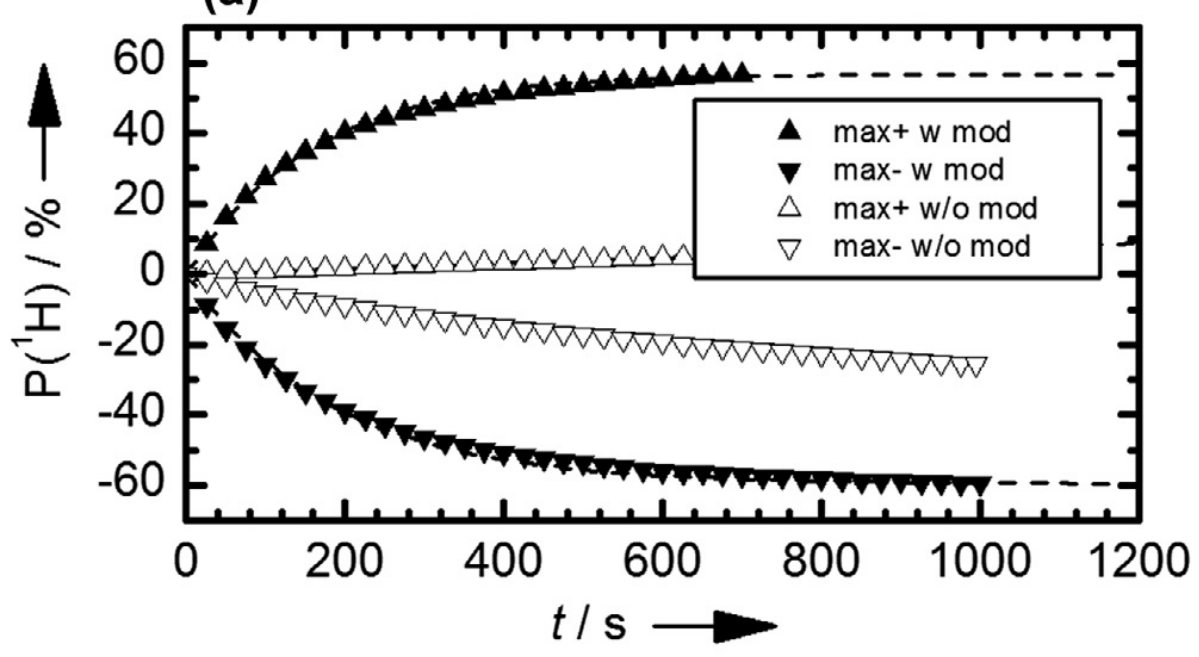

(b)

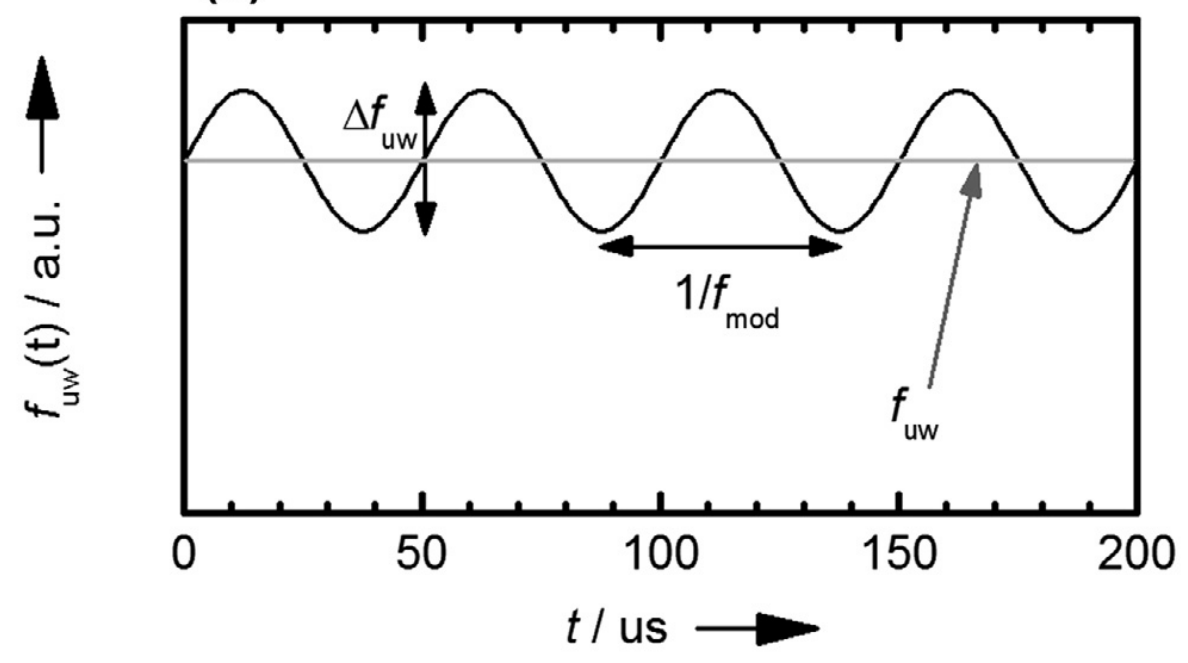

Figure 4: (Top) Negative and positive ${ }^{1} \mathrm{H}$ DNP build-up curves measured at $T=1.2 \mathrm{~K}$ and $B_{0}$ $=6.7 \mathrm{~T}$, with and without frequency modulation (sample: 10:40:50 (v/v/v) $\mathrm{H}_{2} \mathrm{O}: \mathrm{D}_{2} \mathrm{O}:$ glycerol$\mathrm{d}_{8}$ mixture with $25 \mathrm{mM}$ TEMPOL). The optimal frequencies $v_{\mu \mathrm{w}}=187.85$ and $188.3 \mathrm{GHz}$ were set for positive or negative DNP respectively, with a microwave power $P_{\mu \mathrm{w}}=87.5 \mathrm{~mW}$. An amplitude $\Delta v_{\mu \mathrm{w}}=100 \mathrm{MHz}$ was used for frequency modulation. (Bottom) Scheme illustrating the frequency modulation method. The microwave frequency typically varies in a sinusoidal fashion according to $v_{\mu \mathrm{w}}(\mathrm{t})=v_{\mu \mathrm{w}}+1 / 2 \Delta v_{\mu \mathrm{w}} \sin \left(2 \pi v_{\text {mod }} \mathrm{t}\right)$ where $v_{\mu \mathrm{w}}$ is the average frequency, $\Delta v_{\mu \mathrm{w}}$ the amplitude of the frequency modulation, and $v_{\text {mod }}$ the modulation frequency. Copied with permission from [41]. 


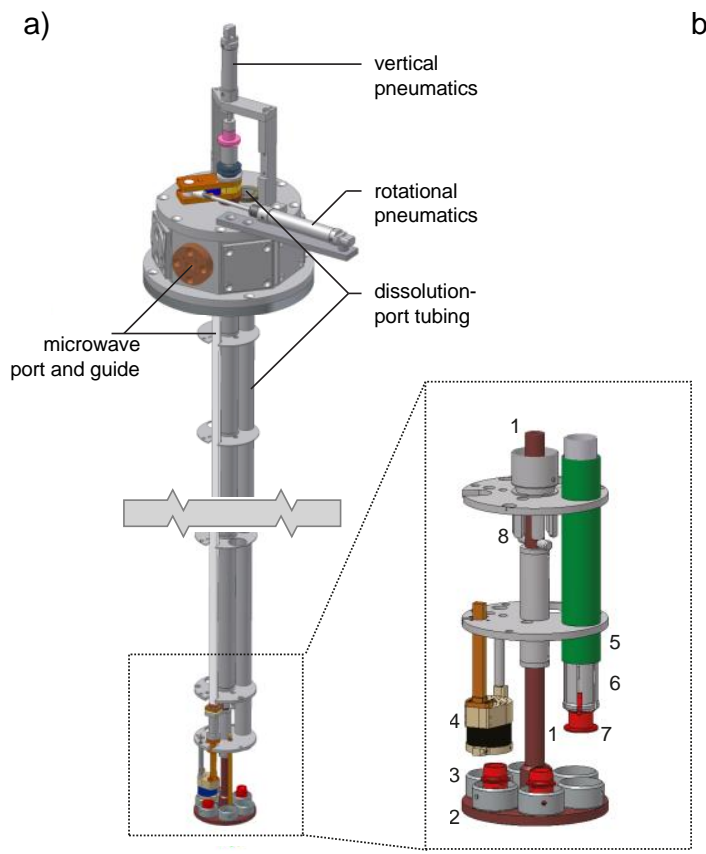

b)

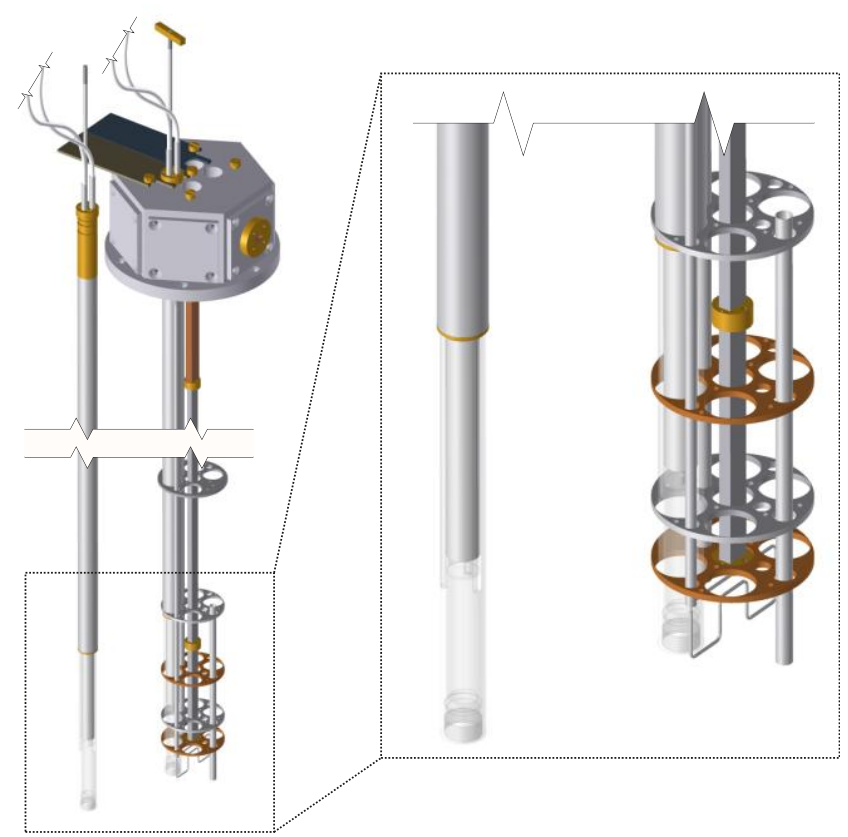

Figure 5: Schematic drawings of two multi-sample dissolution DNP probes. a) Revolver design with six sample holders: The rotationally and vertically movable axis (1) connects the room-temperature space to the sample holder platform (2). Six bottom-closed cylinders (3) can be combined with the top part of the cavity (4) to form the resonant microwave structure. The dissolution port (5) runs from room-temperature to the sample space guiding the grabber (6) to reach the sample cups (7). A pin-in-channel system (8) guides the revolver mechanism to open, rotate, and close to the correct positions. b) Design with four parallel sample inserts that can be polarized and dissolved independently with very short delays between two dissolutions. 


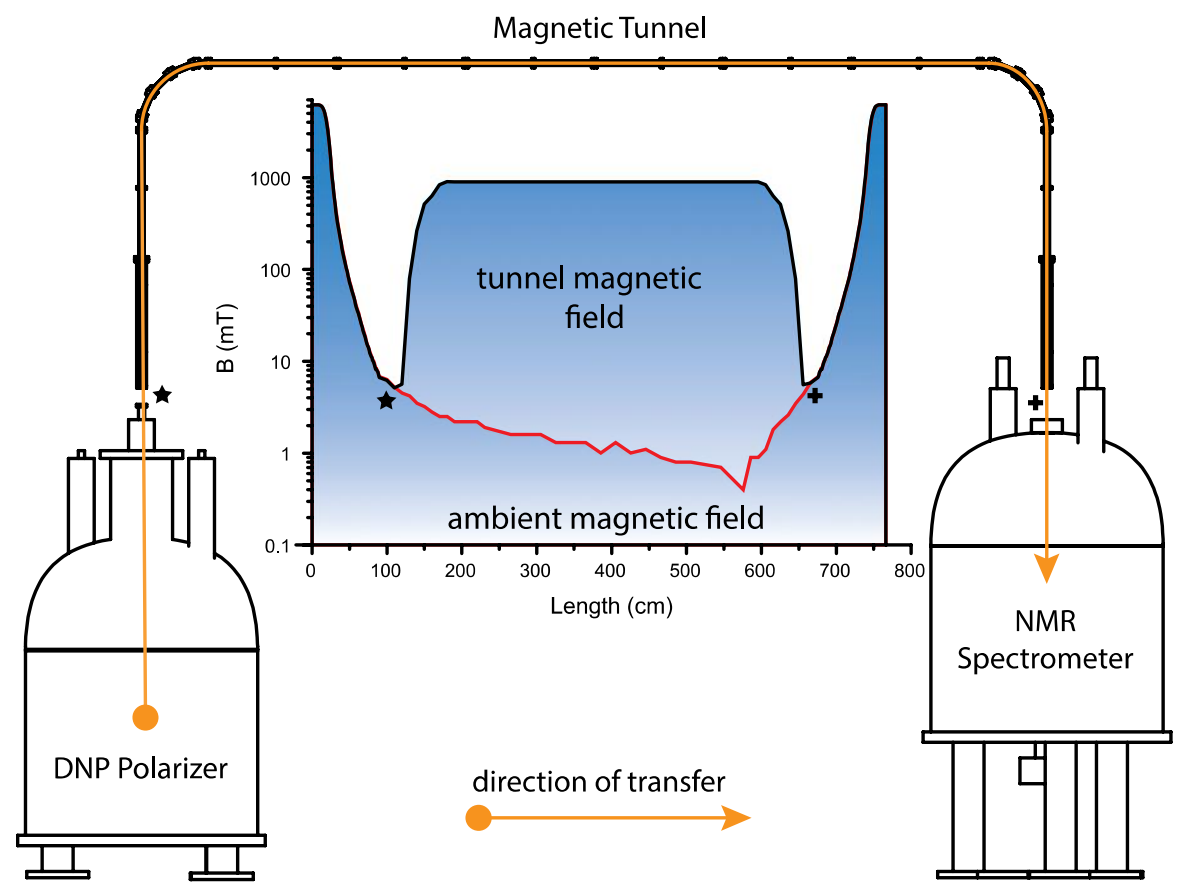

Figure 6: The magnetic field strength in the course of the transfer of the hyperpolarized fluid from the DNP polarizer to the unshielded $300 \mathrm{MHz}$ NMR spectrometer through a magnetic tunnel (black line) or without tunnel (red line). The star and cross indicate the polarizer-tunnel and tunnel-spectrometer junctions. Copied with permission from [51]. 


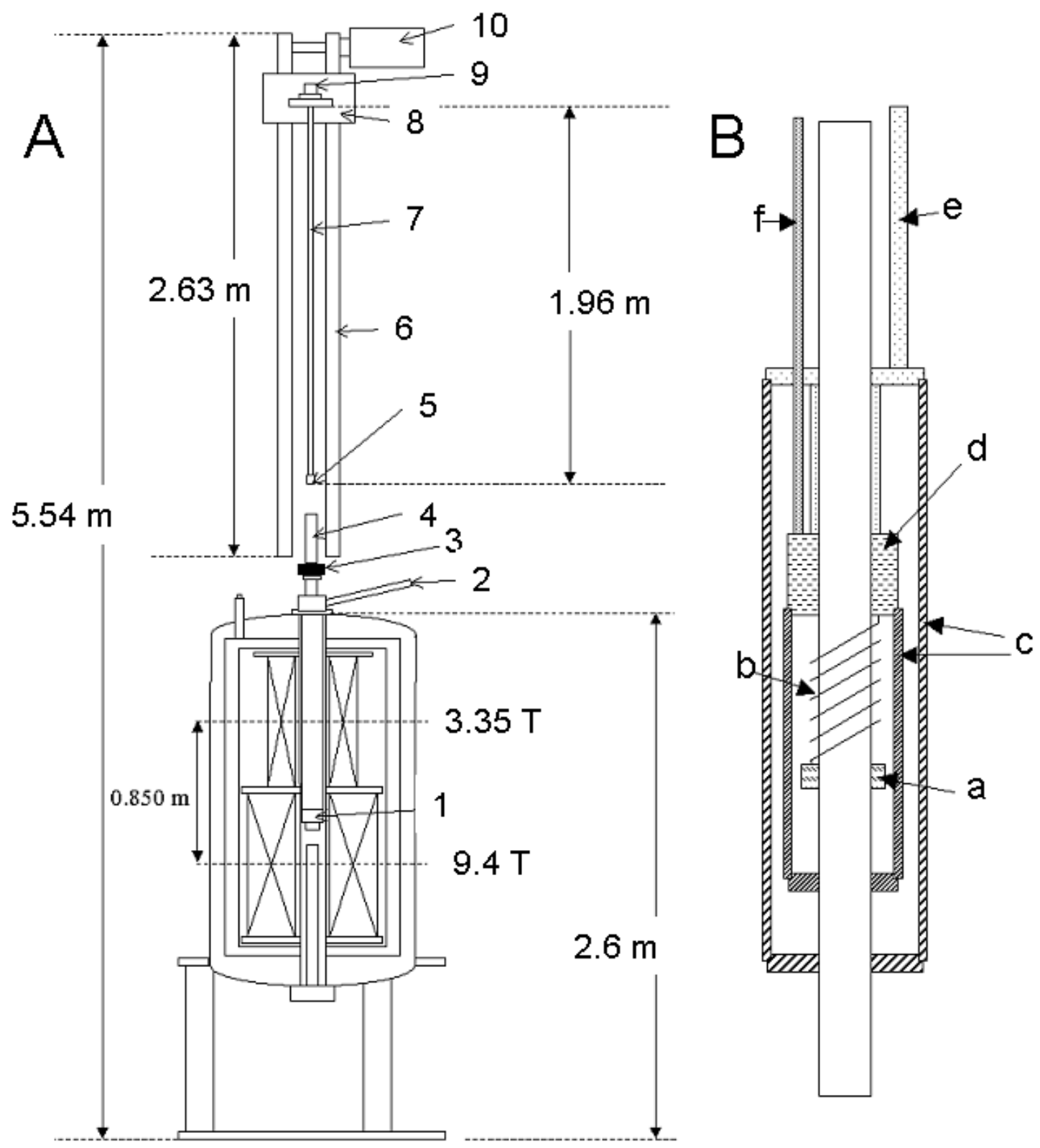

Figure 7: A-Schematic representation of the DNP NMR spectrometer: (1) dissolution dock, (2) helium inlet for cryoinsert, (3) vacuum valve, (4) air lock, (5) sample cup, (6) actuator, (7) waveguide, (8) carriage, (9) Rexolite vacuum cap, (10) stepper motor for actuator control. B-Schematic representation of continuous flow helium cryoinsert: (a) ring with helium inlet to sample space, (b) thin capillary acting as low impedance, (c) thermal shields, (d) reservoir and phase separator, (e) helium outlet, (f) helium inlet. Copied with permission from [59]. 


\section{Tables}

Table 1: Typical Polarization Levels and Build-Up Times

\begin{tabular}{|c|c|c|c|c|c|c|c|c|}
\hline Radical & $\begin{array}{l}c_{\text {rad. }} \\
{[\mathrm{mM}]}\end{array}$ & $\begin{array}{c}\text { Field } \\
{[\mathrm{T}]}\end{array}$ & $\begin{array}{c}T \\
{[\mathrm{~K}]}\end{array}$ & $\begin{array}{l}P\left({ }^{1} \mathrm{H}\right. \\
)[\%]\end{array}$ & $\begin{array}{c}\tau\left({ }^{1} \mathrm{H}\right) \\
{[\mathrm{s}]}\end{array}$ & $\begin{array}{c}P\left({ }^{13} \mathrm{C}\right) \\
{[\%]}\end{array}$ & $\begin{array}{c}\tau\left({ }^{13} \mathrm{C}\right) \\
{[\mathrm{s}]}\end{array}$ & Ref. \\
\hline 4-охо-Тempo & 50 & 6.7 & 1.2 & 91 & - & $71^{*}$ & 1980 & {$[26]$} \\
\hline 4-amino-Tempo & 40 & 7 & 4 & 16.8 & 135 & - & - & {$[68]$} \\
\hline 4-охо-Tempo & 50 & 6.7 & 1.2 & 63.3 & - & - & - & {$[41]$} \\
\hline 4-охо-Tempo & 30 & 3.35 & 1.35 & 30 & 580 & 7.4 & 1014 & {$[37]$} \\
\hline 4-охо-Тетро & 40 & 4.6 & 1.15 & - & - & 18.3 & 1058 & {$[28]$} \\
\hline Tempol & 58 & 7 & 1 & - & - & $35^{\dagger}$ & 2200 & {$[27]$} \\
\hline Trityl OX063 & 15 & 4.64 & 1 & - & - & $64 / 70^{+}$ & 3500 & {$[24]$} \\
\hline Trityl OX063 & 15 & 4.6 & 1.15 & - & - & 54.4 & 3310 & {$[28]$} \\
\hline Trityl OX063 & 15 & 3.35 & 1.35 & - & - & $45^{+}$ & 670 & {$[20]$} \\
\hline Trityl OX063 & 15 & 3.35 & 1 & - & - & 36 & 9500 & {$[22]$} \\
\hline
\end{tabular}

